\title{
Preclinical analysis of human mesenchymal stem cells: tumor tropism and therapeutic efficiency of local HSV-TK suicide gene therapy in glioblastoma
}

\author{
Lasse Dührsen', Sophie Hartfuß', Daniela Hirsch², Sabine Geiger ${ }^{2}$, Cecile L. Mair ${ }^{1}$, \\ Jan Sedlacik ${ }^{3}$, Christine Guenther ${ }^{2}$, Manfred Westphal', Katrin Lamszus', Felix G. \\ Hermann ${ }^{2}$ and Nils Ole Schmidt ${ }^{1}$ \\ 1 Department of Neurosurgery, University Medical Center Hamburg-Eppendorf, 20246 Hamburg, Germany \\ 2 Apceth Biopharma, 81377 Munich, Germany \\ 3 Department of Neuroradiology, University Medical Center Hamburg-Eppendorf, 20246 Hamburg, Germany \\ Correspondence to: Nils Ole Schmidt, email: nschmidt@uke.de \\ Keywords: glioblastoma; brain tumor; stem cells; gene therapy; migration \\ Received: January 27, $2019 \quad$ Accepted: June 19, $2019 \quad$ Published: October 22, 2019 \\ Copyright: Dührsen et al. This is an open-access article distributed under the terms of the Creative Commons Attribution License \\ 3.0 (CC BY 3.0), which permits unrestricted use, distribution, and reproduction in any medium, provided the original author and \\ source are credited.
}

\section{ABSTRACT}

Glioblastoma are highly invasive and associated with limited therapeutic options and a grim prognosis. Using stem cells to extend current therapeutic strategies by targeted drug delivery to infiltrated tumors cells is highly attractive. This study analyzes the tumor homing and therapeutic abilities of clinical grade human mesenchymal stem cells (MSCs) in an orthotopic glioblastoma mouse model. Our time course analysis demonstrated that MSCs display a rapid targeted migration to intracerebral U87 glioma xenografts growing in the contralateral hemisphere within the first $48 \mathrm{~h}$ hours after application as assessed by histology and 7T magnetic resonance imaging. MSCs accumulated predominantly peritumorally but also infiltrated the main tumor mass and targeted distant tumor satellites while no MSCs were found in other regions of the brain. Intratumoral application of MSCs expressing herpes simplex virus thymidine kinase followed by systemic prodrug application of ganciclovir led to a significant tumor growth inhibition of $86 \%$ versus the control groups $(p<0.05)$, which translated in a significant prolonged survival time $(p<0.05)$. This study demonstrates that human MSCs generated according to apceth's GMP process from healthy donors are able to target and provide a significant growth inhibition in a glioblastoma model supporting a potential clinical translation.

\section{INTRODUCTION}

Glioblastoma represent one of the most aggressive brain tumor types with a devastating prognosis of 1215 months after diagnosis despite intensive treatment including surgery, radiation and chemotherapy [1]. Its highly invasive nature within the brain parenchyma limits surgical resection and recurrence due to remaining infiltrating tumors cells is inevitable [2]. The blood-brainbarrier limits available systemic pharmacological options and glioblastoma molecular diversity and instability often results in the rapid development of therapeutic resistance
[3]. The inherent capability of stem and progenitors cells for extensive targeted migration towards brain tumors has the potential to improve drug delivery and to overcome these therapeutic limitations [4].

Preclinical data has demonstrated the efficiency of stem cell based-targeted delivery of various therapeutic payloads to glioblastoma xenografts [5-8]. Clinical proof-of-concept was confirmed in a recently completed safety/feasibility study (NCT01172964) in which a neural stem cell mediated enzyme-prodrug treatment strategy was tested in patients with recurrent glioblastoma [9]. However, one of the major challenges for clinical 
translation of stem cell-based concepts in neurological disease remain the choice of type and origin of the stemor progenitor cells [10]. Neural stem- or progenitor cells of the central nervous system pose logistical and ethical problems especially since they are often of embryonal or fetal origin. Other more easily accessible tissue sources such as the bone marrow would be an ideal source for stem cells. Bone marrow-derived mesenchymal stem cells have been demonstrated to be effective in various preclinical cancer models including glioblastoma suggesting promise for future clinical translation [11].

A recently finished phase I, first in human and first in class trial using genetically modified autologous mesenchymal stem cells expressing herpes simplex virus thymidine kinase (HSV-TK) in combination with ganciclovir (GCV) demonstrated acceptable safety and tolerability in advanced gastrointestinal cancer patients [12]. Therefore, we assessed the migratory and tumor homing capabilities of these genetically modified human mesenchymal stem cells in an intracerebral glioblastoma model and evaluated the therapeutic efficiency of local HSV-TK suicide gene therapy.

\section{RESULTS}

\section{Characterization of human mesenchymal stem cells and transgene expression}

Human MSCs were isolated from bone marrow of healthy donors in accordance with apceth's GMP process [12]. The isolated MSCs were retrovirally transduced with therapeutic vector that constitutively expresses HSV-TK under control of the EFS promoter for in vitro and in vivo efficacy. Furthermore, cells were transduced with a GFP encoding vector to allow for in vivo and ex vivo tracking of GFP expressing cells. Subsequently, the transduced cells were purified using puromycin selection, expanded and cryo-preserved.

To ensure an MSC-like identity, the cells were characterized in regards to differentiation capacity, the expression of surface markers and transgene expression. The genetically modified MSCs differentiated into adipocytes and osteocytes (Figure 1A). Both, GFP and HSV-TK expressing MSCs were positive ( $>94 \%$ ) for MSC markers CD73 (100.0\%, 99.8\%), CD90 (94.5\%, $99.9 \%)$ and CD105 $(99.3 \%, 98.7 \%)$ and negative (< $2 \%)$ for impurity markers CD19 $(0.7 \%, 0.6 \%)$, CD34 $(0.4 \%, 1.0 \%)$ and CD45 $(0.3 \%, 1.2 \%)$ as well as HLADR $(0.6 \%, 0.6 \%)$ (Figure 1B). Flow cytometric analysis revealed $10.6 \%$ GFP positive MSC after transduction and $99.2 \%$ positive cells after selection. For HSV-TK expressing MSCs, $24.2 \%$ of cells were positive before and $99.6 \%$ after puromycin selection as determined using an antibody directed to the hemagglutinin-tag (HA-tag) linked to the HSV-TK transgene (Figure 1C,1D). After thawing 96.15\% (MSC-GFP) and 97.69\% (MSC-TK) of cells were vital, respectively as determined by Annexin V/7AAD flow cytometry.

\section{In vitro bystander killing depends on gap junctions}

Cell that are transduced with HSV-TK are efficiently killed by GCV. The bystander-killing refers to the fact that nearby non-transduced cells are also sensitive towards GCV treatment. It has previously been shown that gap junctions are necessary to allow efficient distribution of phosphorylated GCV between cells, which is a prerequisite for the bystander effect $[13,14]$.

A dye transfer assay was performed to demonstrate gap junction formation between MSC_HSV-TK and different glioblastoma cell lines (U87, G55T2 and GL261). Efficient transfer of gap junction permeable dye Calcein AM to CMTPX (cell tracker red) negative tumor cells $4 \mathrm{~h}$ after coculture (U87 97.9+/-0.0\%, G55T2 $86.2+/-1.2 \%$, GL261 37.0+/-1.7\% Calcein positive tumor cells) was observed which could be inhibited by gap junction inhibitor Carbenoxolone (Figure 2A). It was further confirmed, that the dye transfer is cellcell contact dependent, since no dye transfer was observed when cells were separated by transwells (Figure 2B).

To demonstrate that genetically modified MSCs that constitutively express HSV-TK are able to kill glioblastoma cells in the presence of $\mathrm{GCV}$, in vitro bystander killing assays were performed. MSC_HSVTK were cocultured with CMFDA (cell tracker green) or GFP-labeled U87, G55T2 or GL261 tumor cells at a ratio of $1: 1$. The cocultures were treated with GCV for three consecutive days before quantitative analysis by flow cytometry to determine the percentage of surviving tumor cells. According to the FACS data obtained, a significant reduction of surviving tumor cells was observed after coculture of HSV-TK expressing MSCs with CMFDA stained U87, G55T2 or GL261 glioblastoma cells and addition of GCV $(21.2+/-1.0 \%, 16.8+/-0.8 \%$ or $11.0+/-$ $0.1 \%$ surviving tumor cells, respectively) compared to control samples without GCV treatment (Figure 2C). A reduced percentage of vital tumor cells was also observed for U87 and GL261 control samples (without MSC coculture) and the addition of GCV, indicating slightly toxic effects of GCV treatments on these tumor cells $(77.9+/-4.9 \%$ or $77.5+/-8.0 \%$ surviving tumor cells, respectively) (Figure 2C). Lower M:T ratios, up to 1:100, were observed to induce efficient killing of U87 target cells after addition of GCV prodrug (1:10 23.7+/-2.0\%, $1: 5023.3+/-1.8 \%, 1: 100 \quad 31.7+/-3.3 \%$ surviving U87 cells). Hence, the in vitro data indicate an efficient antitumoral effect of MSC_HSV-TK against glioblastoma cells, even at low M:T ratios (Figure 2D). 


\section{MSC display tumor targeted migration in vitro}

MSCs are useful vehicles for the delivery of therapeutic genes to tumors because of their ability to specifically migrate towards tumor tissue [11]. To confirm
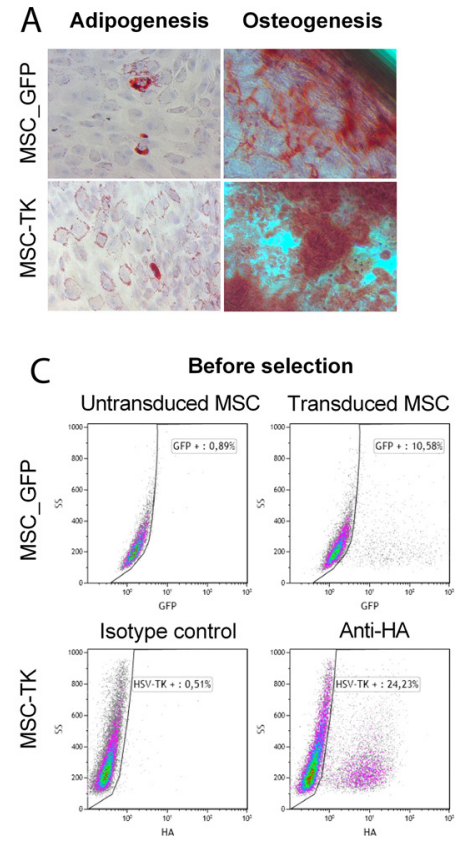

this tumor tropism in vitro, a modified Boyden chamber assay was performed. Conditioned medium collected from different glioblastoma cells (G55T2, U87 and G1261) was used as an attractant of MSC. Compared to unconditioned medium (40+/-6 migrating cells), conditioned medium

\section{B}

\begin{tabular}{|l|r|r|}
\hline & MSC_GFP (\%) & MSC-TK (\%) \\
\hline CD73 & 100.0 & 99.8 \\
\hline CD90 & 94.5 & 99.9 \\
\hline CD105 & 99.3 & 98.7 \\
HLA-DR & 0.6 & 0.6 \\
\hline CD19 & 0.7 & 0.6 \\
\hline CD34 & 0.4 & 1.0 \\
\hline CD45 & 0.3 & 1.2 \\
\hline
\end{tabular}

D After selection

Untransduced MSC Transduced MSC
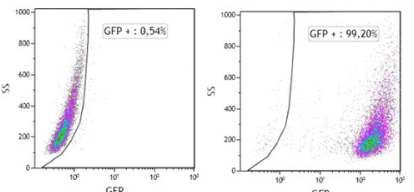

Isotype control

Anti-HA
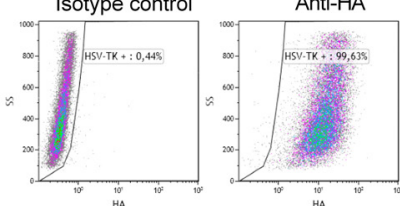

Figure 1: Characterization of transduced MSCs by in vitro differentiation assay and flow cytometry. The capacity of genetically modified MSCs to differentiate to adipocytes and osteocytes was confirmed by differentiation assays (A). Percentage of positive surface marker. MSC_GFP and MSC-TK were positive for the MSC markers CD73, CD90 and CD105 and negative for the impurity markers tested (CD19, CD34 and CD45) (B). After transduction with retroviral vectors to express GFP or HSV-TK, 10.6 and 24.2\% of cells were transduced before and 99.2 and $99.6 \%$ of cells after puromycin selection, respectively (C and $\mathbf{D})$. Abbreviation: HA, hemagglutinin.
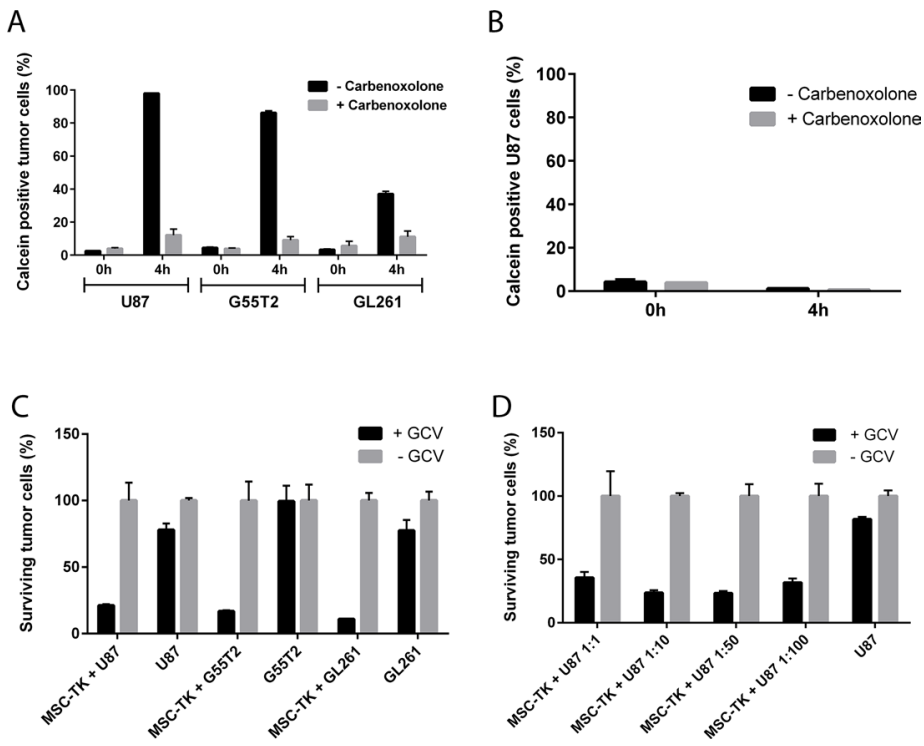

Figure 2: In vitro gap junction formation and bystander killing of glioblastoma cells by HSV-TK expressing MSCs. Dye transfer of Calcein stained MSCs to glioblastoma cells indicate efficient gap junction formation (A and B). Anti-tumoral efficacy was demonstrated by significant reduction of surviving U87, G55T2 and GL261 tumor cells after MSC-HSV-TK coculture and GCV cotreatment (C) even with at low M:T ratios up to 1:100 (D). 
collected from G55T2, G1261 and U87 led to a 7.4-fold (295+/-42 migrating cells, $\mathrm{p}<0.01)$, 7-fold $(281+/-14$ migrating cells, $\mathrm{p}<0.01)$ and 4.9-fold $(195+/-7$ migrating cells, $\mathrm{p}<0.01$ ) increase in migration, respectively (Figure $3 \mathrm{~A})$. In addition, the human neural stem cell line HB1. F3 was assessed in the modified Boyden chamber assay. Compared to the basal migration rate of $46+/-21$ cells induced by unconditioned medium, medium collected from G55T2, G1261 and U87 glioblastoma increased migration 5-fold $(226+/-20, \mathrm{p}<0.01), 4.7$-fold $(216+/-22$, $\mathrm{p}<0.01)$ and 3.7-fold $(171+/-20, \mathrm{p}<0.01)$, respectively (Figure 3B). HB1.F3 has been previously described as a cell line that very efficiently homes to glioblastoma xenografts $[15,16]$. Compared to the results achieved with HB1.F3, the human MSC demonstrated a similar pattern for a targeted migration towards glioblastoma.

\section{Human MSC efficiently target glioblastoma xenografts after intracranial injection}

The next aim was to confirm the tumor tropism of human MSCs in an in vivo model. Human U87 glioblastoma cells were injected into the right forebrain of mice to establish tumors. Ten days later, $5 \times 10^{4}$ DiIlabeled MSCs from cell culture were injected into the left forebrain using the matching contralateral coordinates as used for the tumor induction ( $\mathrm{n}=5$ mice). Seven days later, histological analysis demonstrated a directed migration of MSCs from the injection site via the corpus callosum towards the tumor in the contralateral hemisphere. DiIpositive MSCs were found preferably at the tumorparenchyma border encircling the tumor but also invading into the tumor mass. Serial sections of the whole brain did not reveal any DiI-positive MSC in other distant cerebral or cerebellar regions confirming a targeted tumor tropism (Figure 4).

In order to assess the spatial and temporal dynamics of MSC migration, we performed a histological time course analysis. Ten days after U87 human glioblastoma cell injection into the right forebrain, 15 mice received a stereotactic injection of $2 \times 10^{5}$ freshly thawed eGFPexpressing MSCs to the contralateral hemisphere. Groups of mice $(\mathrm{n}=5$ each) were sacrificed 2, 3 and 7 days after MSC application and serial sections of the whole brain were histologically evaluated to detect MSCs (Figure 5A). Within the first two days MSCs were able to reach the tumor in the contralateral hemisphere, migrated into the tumor mass (Figure 5B) and accumulated in the peritumoral area directly at the brain parenchyma-tumor border (Figure 5C). Even a small tumor satellite distant from the main tumor mass was targeted by the MSCs (Figure 5D). Quantification of migrated GFP-expressing MSCs revealed that the tumor homing occurred within the first two days with the majority of cells localized peritumorally (PT) when compared to intratumorally (IT) localized GFP-positive cells (PT 25+-2.9 cells/hpf vs IT 9.5+- 1.3 cells/hpf, $\mathrm{p}<0.001 \mathrm{MWU}-$ test)(Figure 5E). The number of IT- or PT-localized MSCs was not significantly different between day 2 and day 7 (d2 IT 9.5+-1.3 cells/hpf vs d7 IT 6+-0.9, p=0.074; d2 PT 25+-2.9 cells/hpf vs d7 PT 20+-1.4, $\mathrm{p}=0.12$ ). The results demonstrate that MSCs are able to migrate in a targeted manner to glioblastoma and small tumor satellites distant from the main tumor mass making them ideal delivery vehicles for therapeutic genes.

\section{MSC migration can be tracked in vivo by non- invasive MR imaging}

With regard to future clinical development of MSCbased products, clinical applicable non-invasive methods for monitoring distribution of cellular therapeutics in the patients are highly desirable. Therefore, we tested if MR imaging is a suitable method to allow in vivo tracking of MSCs, which have been loaded with a superparamagnetic iron oxide (SPIO) based MRI tracer. Tumors were established as described above. The GFP-expressing L87 MSC cell line loaded with SPIO or without SPIO as controls were injected into the left forebrain at the same location contralateral to the growing tumor $(n=6)$. MR
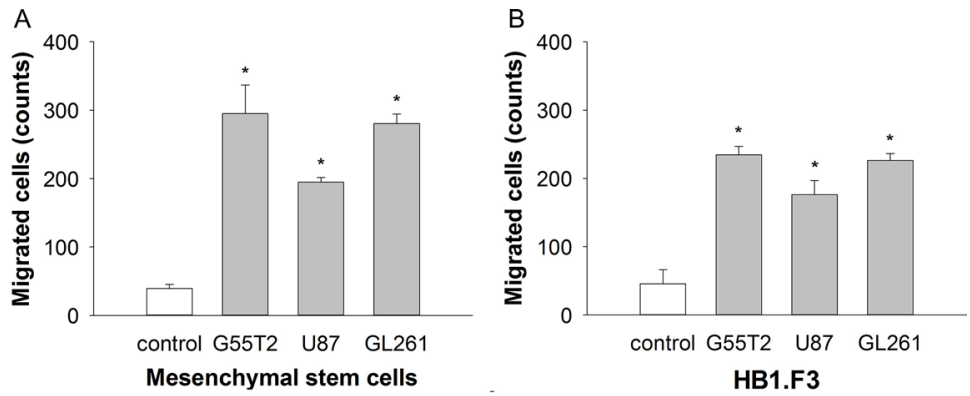

Figure 3: Mesenchymal stem cell (MSC) migration (A) in response to conditioned media from glioblastoma cell lines in a 96-well modified Boyden chamber assay in comparison to the well-established human neural stem cell line HB1.F3 (B). Conditioned media from all tumor cell lines significantly stimulated the directional migration of the MSCs compared to the control ( ${ }^{*} \mathrm{p}<0.001, \mathrm{t}$ test $)$. 
imaging at day 1, 2, 5 and 7 demonstrated that the MSCs reached the tumor area in the contralateral hemisphere already within the first 24 hours (Figure 6). Hypointensive signals in axial SWI across the corpus callosum (Figure $6 \mathrm{~B})$ at day 1 indicated a directed migration towards the tumor. In coronal sections (Figure 6C) the hypointensive signals within the tumor area further increased from day 1 to day 7 indicating that further SPIO-loaded MSC reached the tumor. In the control animals with MSCs but without SPIOs, no similar signal hypointensities were observed (Figure 6D, 6E, 6F). MSCs enriched in the tumor which had spread into the ventricular system as indicated by the signal hypointensities in the midline (Figure 6B, 6C arrowhead). This was confirmed by Prussian blue staining demonstrating blue colored SPIO-loaded MSC within the tumor (Figure 6G). Furthermore, histological sections after day 7 confirmed the presence of GFP-labeled MSCs within the tumor area (Figure 6H). Histological quantification of GFP-positive MSCs within the tumor and the peritumoral area confirmed a similar migration pattern and no significant impact of SPIO loading on the migratory capabilities (Figure 6I). The results indicate that MR imaging is a potential clinically applicable method to monitor biodistribution of MSC-based therapeutics.

\section{Therapeutic efficiency of HSV-TK expressing MSC on glioblastoma growth and survival}

After tumor specific homing of MSCs in vivo had been established, our aim was to assess the therapeutic efficiency of HSV-TK expressing MSC in an in vivo glioblastoma model. For this U87 glioblastoma xenografts were established by stereotactic injection into the right forebrain of 6-week old nude mice as described above. Local intratumoral injection of $0.4 \times 10^{6} \mathrm{MSC}_{-} \mathrm{TK}$ followed by five days of systemic GCV administration led to a significant tumor growth inhibition of $86 \%$ and $77 \%$ compared to the control groups (MSC-TK(-) and $\mathrm{NaCl}(+)$ ), respectively as assessed by MR imaging at day 20 after tumor cell administration (Figure 7A). Animals who received HSV-TK expressing MSCs followed by GCV administration displayed only small tumors and in five animals hardly any tumor mass was visible (Figure 7B). 3D-quantification based on T2 MR imaging confirmed significant smaller tumor volumes $(339.5+/-$ $275 \mathrm{~mm}^{3}$ ) than in the controls that received no GCV after MSC-TK injection $\left(2353+/-950 \mathrm{~mm}^{3}\right)$ or that received no cells but GCV $\left(1449+/-331 \mathrm{~mm}^{3}\right)$. This therapeutic effect was paralleled by significant higher bodyweights than in
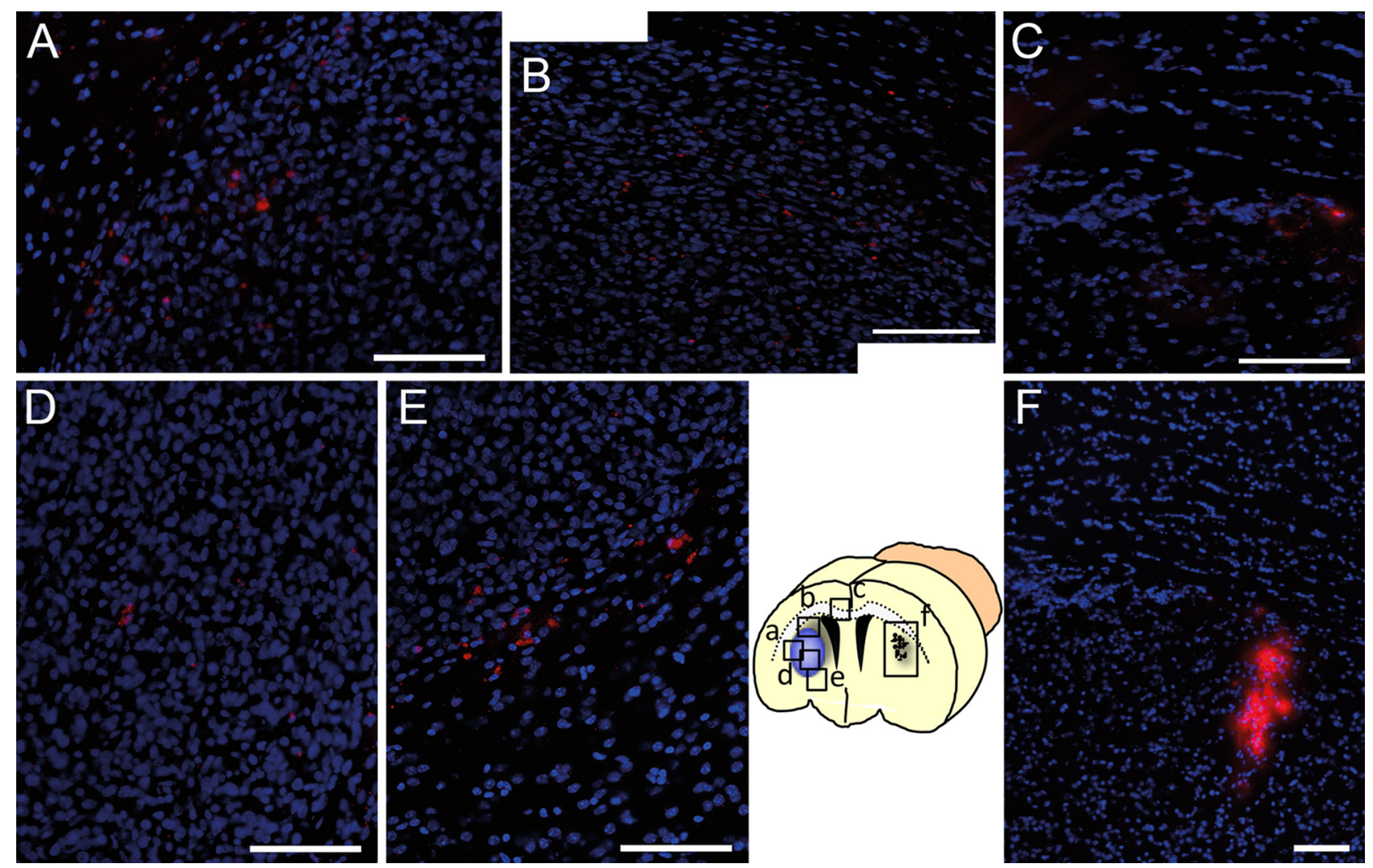

Figure 4: Tumor targeted in vivo migration of DiI-labeled MSCs (red) seven days after stereotactic injection into the contralateral hemisphere (F, injection site) of a growing U87 glioblastoma xenograft. Within seven days DiI-MSCs were found in the tumor area (A and $\mathbf{B})$ after migration towards the glioblastoma in the contralateral hemisphere via the corpus callosum (C). Even in distant parts of the tumor DiI-labeled MSCs were found intratumorally (D) or peritumorally (E) whereas no MSCs were found in other regions of the brain. Scale Bars: $100 \mu \mathrm{m}$. 
the controls that presented rapid tumor growth (Figure 7C). Tumor growth inhibition translated into a significant extend of survival $(\mathrm{p}<0.05)$ with a median survival of 29 days compared to the control groups that received MSC-
TK followed by GCV administration and those without cells but GCV (23 days and 25 days, respectively) (Figure 7D).
A
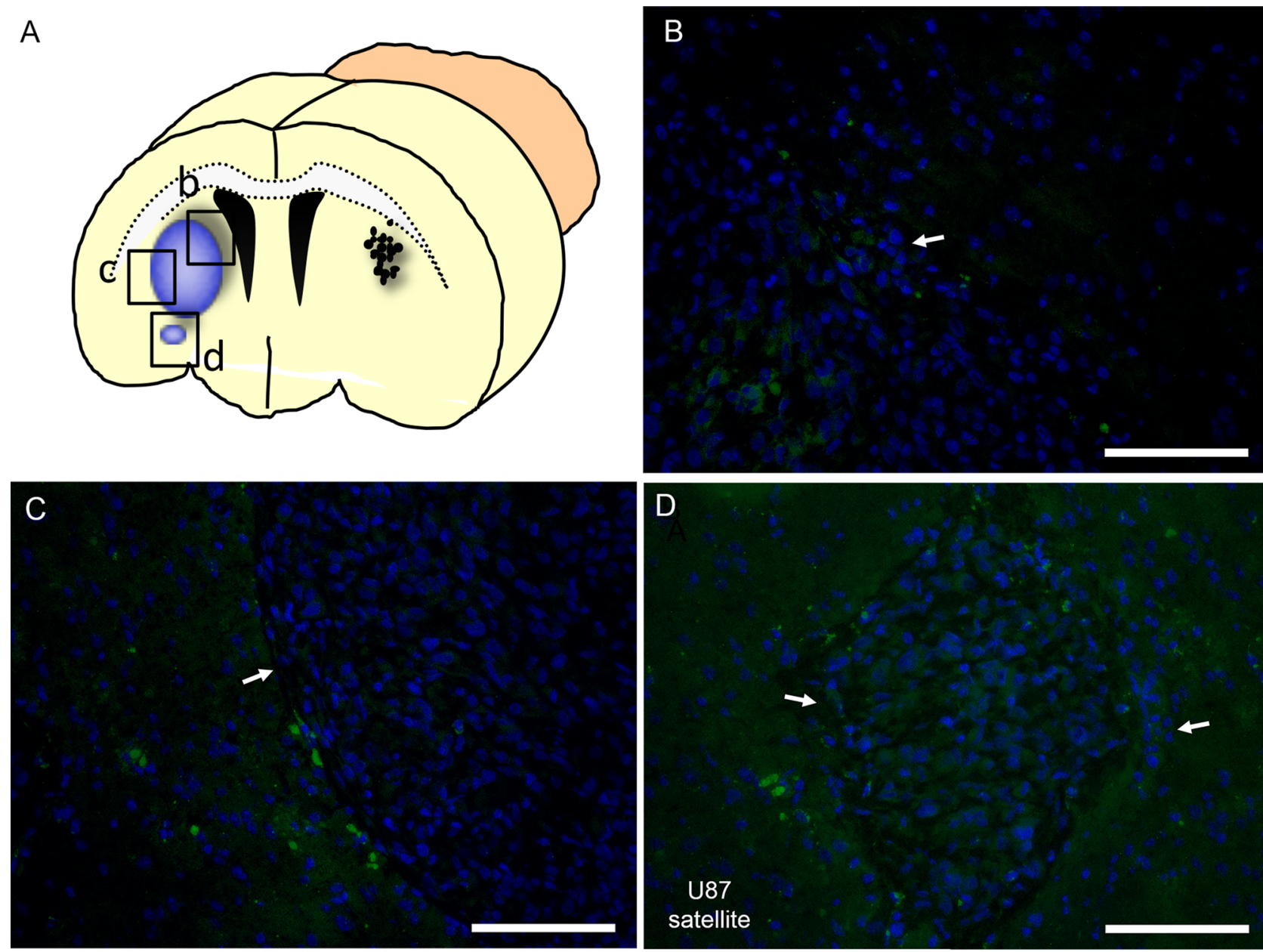

E

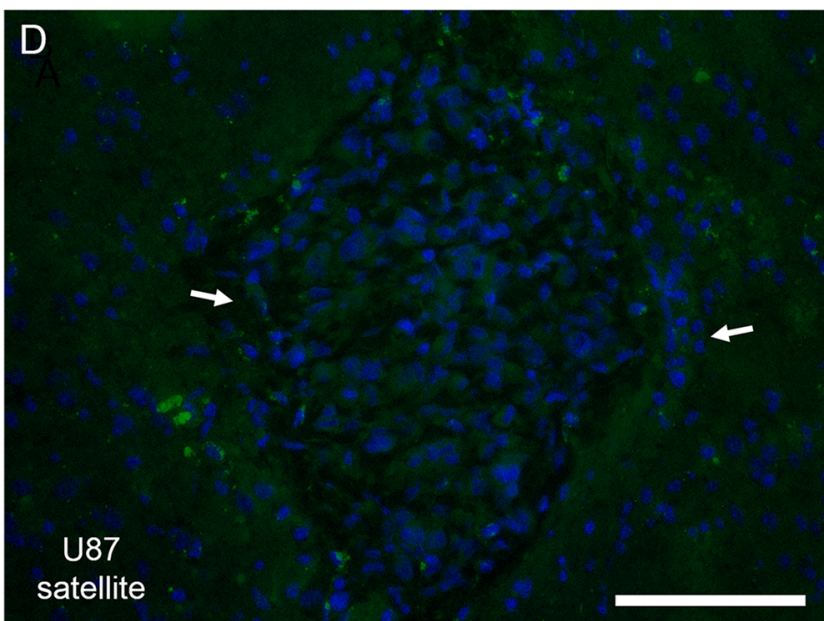

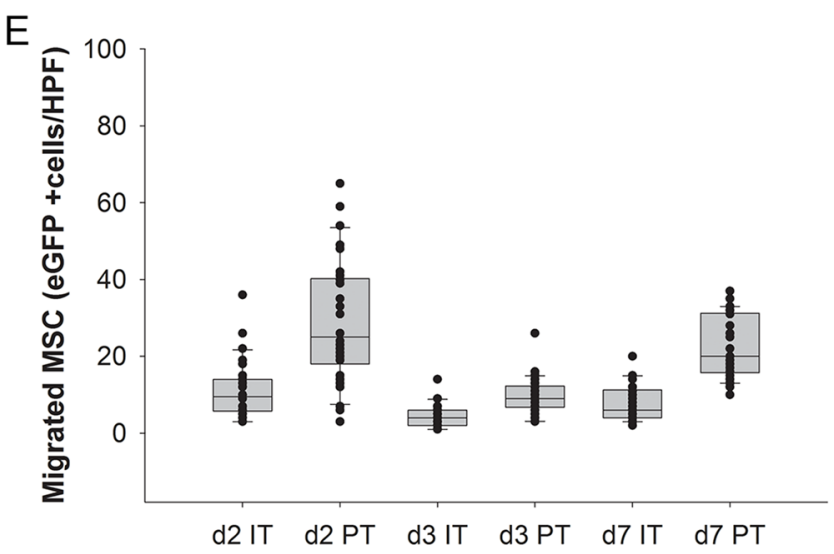

Figure 5: Time course analysis of intratumoral (IT) and peritumoral (PT) accumulation in the intracerebral human U87 glioblastoma model after injection of eGFP-expressing MSCs (green) into the contralateral hemisphere (A). Two days after injection MSCs enriched at the tumor site with intratumoral (B) and peritumoral (C) hot spots. Even a tumor satellite distant from the main tumor mass was peritumorally encircled by MSCs (D). The majority of MSCs was found in the immediate peritumoral area after two days (E, box whisker chart). Arrows = tumor border. Scale Bars: $100 \mu \mathrm{m}$. 


\section{DISCUSSION}

Malignant gliomas are highly invasive and associated with a grim prognosis. Using stem cells to extend current therapeutic strategies by targeted and localized drug delivery to infiltrated tumors cells is highly attractive and furthermore allows the use of additional therapeutic payloads otherwise not applicable by
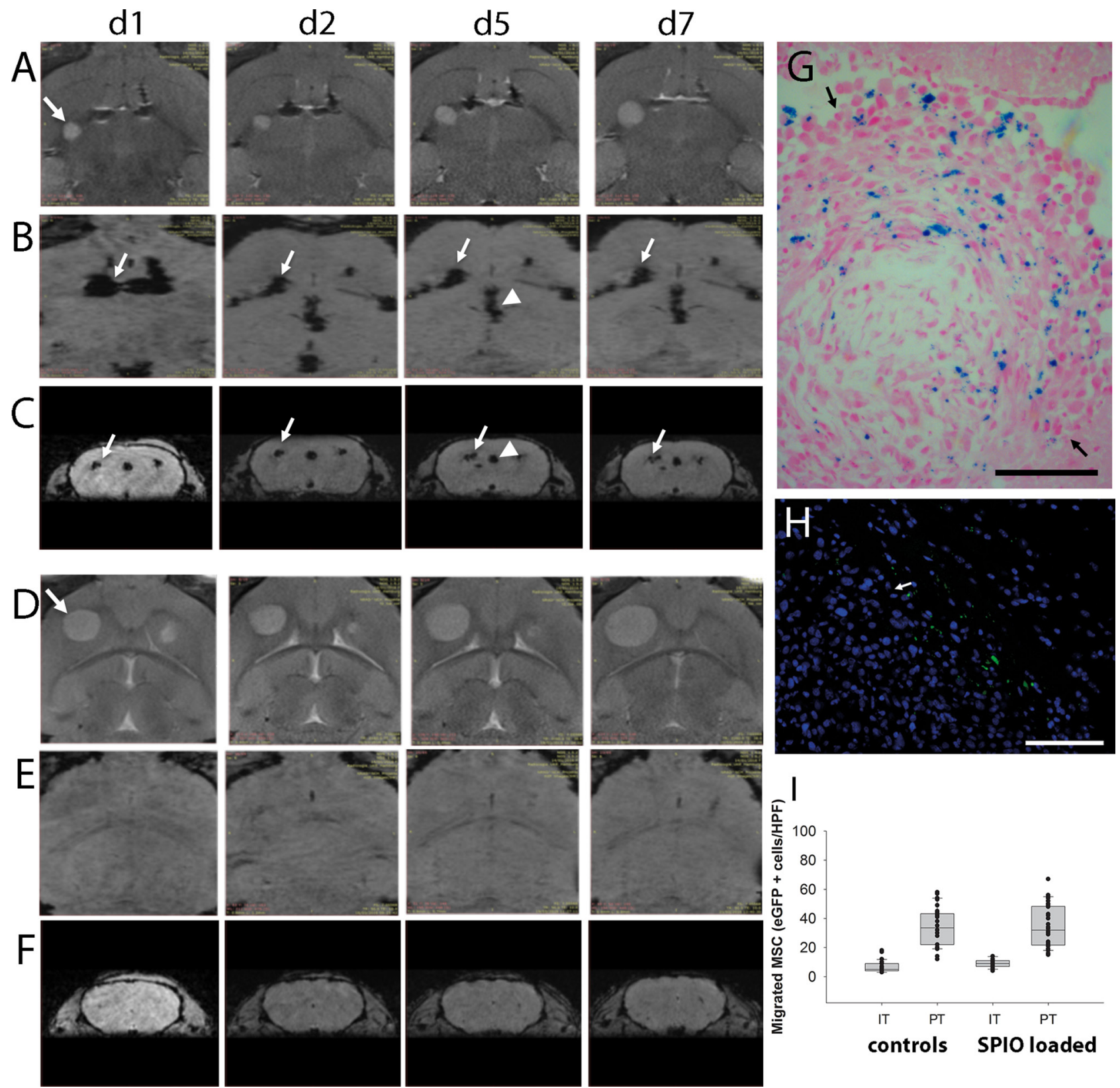

controls SPIO loaded

Figure 6: In vivo MR-imaging of tumor targeted migration of human MSCs towards intracerebral U87 glioblastoma xenografts (A-C = SPIO-loaded MSCs, D-F = control animals with non-loaded MSCs). Axial sections of T2-weighted MR images demonstrate glioblastoma growth (arrow) from day one (d1) to day seven (d7) after MSC injection into the contralateral hemisphere (A, d1-d7; D, d1-d7). Signal voids in axial (B) and in coronal (C) sections of SW images indicated SPIO-labeled MSCs migrating from the injection site (arrows) via the corpus callosum towards the tumor site over time. Signal voids in the ventricular system (arrowhead, e.g. $\mathrm{D} / \mathrm{d} 5$ and $\mathrm{C} / \mathrm{d} 5$ ) indicated MSC presence and (G) histological analysis confirmed tumor spread into the ventricular system where presence of SPIO-loaded MSCs within the tumor mass (arrows) was confirmed by Prussian blue staining (blue). (E, F) Corresponding SW images of control mice which received MSC without SPIO loading did not display similar signal voids. (H) Tumor tropism of human SPIOloaded MSCs was furthermore confirmed by the presence of GFP-positive MSCs at the contralateral tumor site (arrow = tumor border). (I) Histological quantification of SPIO-loaded and non-loaded GFP-positive MSCs within the tumor and the peritumoral area. Scale Bars: $100 \mu \mathrm{m}$. 
conventional delivery strategies [17]. This study analyzes the tumor homing abilities of human MSCs, produced according to apceth's GMP process, in an orthotopic glioblastoma mouse model in preparation for potential clinical translation of this concept.

Our data demonstrated a rapid and targeted migration of intracerebrally injected MSCs towards glioblastoma xenografts growing in the contralateral hemisphere as demonstrated by histological and MR imaging-based analyses. Already within 48 hours, MSCs were found to accumulate into the tumor area even in the most distant parts of the tumor and in small distant tumor satellites, whereas no MSCs were found in other regions of the brain. This is in line with a previous study demonstrating that MSCs displayed a targeted migration towards tumors [8]. Migration of stem- or progenitor cells towards single or multifocal areas of neoplastic, ischemic, degenerative or traumatic lesions within the brain have been observed in a variety of animal models [4, 18]. A number of cytokines and its corresponding receptors such as vascular endothelial growth factor (VEGF) [15, 19], stem cell factor/c-kit [20], stromal cell-derived factor-1/ CXCR4 [8] or platelet derived growth factor-BB [8] have
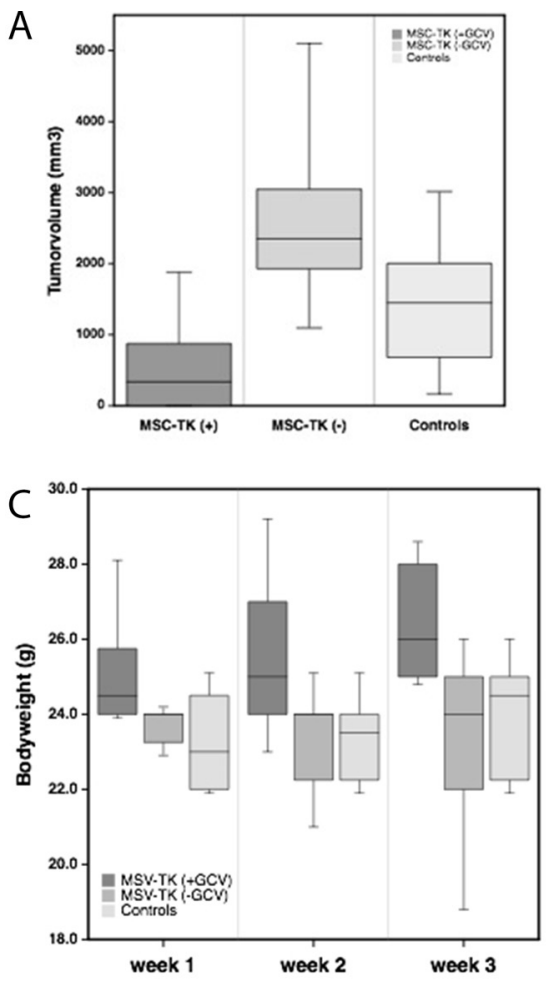

been identified as chemotactic factors for neural stem cells (NSCs) and MSCs. The extensive upregulation of VEGF in malignant gliomas was able to induce a tumor-targeted and long-range migration of NSCs after intracerebral injection $[15,21]$. VEGF demonstrated also significant pro-migratory and pro-invasive effects on human MSCs in a glioma spheroid model [19] but failed to show a similar effect in a Matrigel invasion assay [8]. However, as VEGF is the known major player of angiogenesis in health and disease it also stimulates cerebral endothelial cells to express a multitude of other chemotactic factors potentially guiding stem- and /progenitor cells towards a neuro-pathology [21]. MSCs are able cross the bloodbrain-barrier and to enter the brain after direct venous or arterial intravascular injection [8, 22]. Even after noninvasive intranasal delivery MSCs are able to enter the brain via the olfactory mucosa/cribriform plate and migrate towards gliomas [23] as it has been shown with NSCs [7]. The recently discovered meningeal lymphatic system adds to the complexity of potential pathways in and out of the brain and future studies need to unravel its relevance for cell-based therapies in neurological disorders [24].
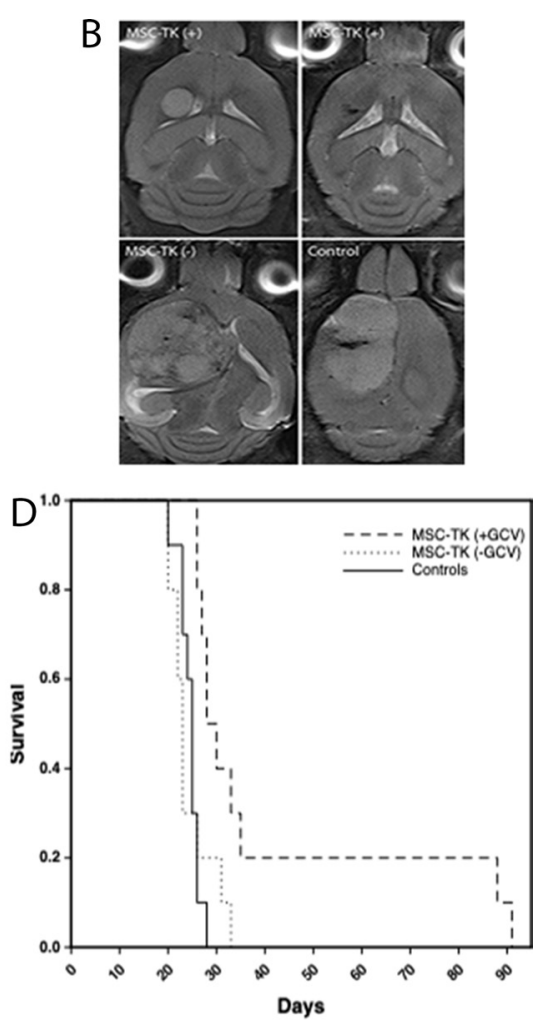

Figure 7: Therapeutic effects of local intratumoral injection of HSV-TK expressing MSC (MSC-TK) followed by five days of systemic GCV administration. (A) In vivo MR-imaging at day 20 after tumor induction demonstrated significant smaller tumor volumes compared to the control groups $(\mathrm{p}<0.05)$. There was no significant difference between the control groups. $(\mathbf{B})$ Representative axial T2-weighted MR images of the different treatment groups. (C) The body weight of animals treated with MSC-TK followed by systemic application of the prodrug GCV were significantly higher than compared to the controls (Box whisker charts, $\mathrm{p}<0.05$ ). (D) Kaplan-Meier analysis of survival with significantly longer survival of treated animals compared to controls ( $\mathrm{p}<0.05$ ). 
Our time course analysis demonstrated that the majority of MSCs were localized peritumorally, surrounding the tumors and that the peri- and intratumoral enrichment occurred within the first two days after intracerebral MSC injection. We observed a stable number of MSCs within the tumor region up to day 7 , but no further increase of MSC density, which is in agreement with Huang et al. using rodent MSCs in a brain stem tumor model [25]. The in vitro analysis comparing the tumor targeted migration of MSCs with the neural stem cell line HB1.F3, which was recently tested in a clinical phase I study in recurrent glioblastoma, revealed a similar tumor tropism towards different glioblastoma cell lines [9, 16]. These findings are in line with a previous comparison of MSCs with the HB1.F3 cell line in a brain stem tumor model [26]. MSCs are multipotent, fibroblast-like cells which can be generated from different tissues including fat, cord blood and bone marrow and easily expanded in culture [27-29]. Their low immunogenic characteristics allow the generation of cell products for allogeneic application [30]. Taken together, these results support the potential clinical use of MSCs as an ethical and logistic ideal alternative to neural stem cells as drug delivery vehicles for the treatment of glioblastoma.

As every novel therapeutic option needs to get integrated in the current standard therapeutic regimen it seems optimal to initiate a stem cell-based therapy in glioblastoma patients directly after resection of the tumor mass. With the number of tumor cells at its minimum, the remaining infiltrated glioma cells can then be targeted by MSCs delivering a therapeutic payload. In this study, we used the well-known HSV-TK suicide gene therapy as a therapeutic paradigm for the MSC-based therapy. The single intratumoral injection of $0.4 \times 10^{6} \mathrm{HSV}-\mathrm{TK}$ expressing human bone marrow-derived MSCs followed by five days of systemic GCV administration led to a significant tumor growth inhibition of $86 \%$ and prolonged survival in the orthotopic U87 glioblastoma mouse model. This is in line with a previous study using HSVTK expressing MSCs derived from adipose tissue, which demonstrated a significant tumor growth inhibition in the U87 glioblastoma model. However, the treatment effects were only assessed early after MSC treatment and the effects on survival were not investigated [29]. Our vitro data using different glioblastoma cells lines demonstrated that gap junction intercellular communication of the HSV-TK expressing MSCs contributed to the significant bystander killing of tumor cells as demonstrated previously by Matuskova et al. [14].

The HSV-TK suicide gene therapy was already clinically tested in a randomized, open-label, phase 3 trial in patients with glioblastoma [31]. However, in this trial the suicide gene therapy was mediated by an adenovirus which is not motile and therefore clearly limiting the therapeutic distribution. Although some therapeutic effects were observed, the clinical trial failed as the primary endpoint was not reached [31]. It seems reasonable to speculate that the motile abilities of HSVTK expressing MSCs have the potential to optimize the therapeutic distribution and may therefore improve therapeutic efficiency. Nevertheless, prodrug-based suicide gene therapies may not be sufficient enough to provide a sustained tumor control in the brain as the therapeutic efficiency also depends on the distribution and concentration of the systemically applied prodrug. Other gene products with a direct therapeutic effect such as TRAIL [32] or interleukin-12 [33] or the simultaneous expression of different therapeutic gene products [34] have been preclinically tested using stem cell-based approaches and may be more effective while also expanding the so far highly limited therapeutic options in malignant glioma.

Our study establishes that our genetically modified human MSCs generated according to apceth's GMP process from healthy donors are able to target and provide a significant growth inhibition and increase survival in a glioblastoma model. Our data support a potential clinical translation as motile MSCs represent an ideal platform technology for the targeted delivery of various therapeutic gene products in malignant glioma.

\section{MATERIALS AND METHODS}

\section{Generation and characterization of human mesenchymal stem cells}

Human MSCs were isolated from bone marrow of healthy donors in accordance with apceth's GMP process using apceth's proprietary medium. Cells were detached using TrypLE Select (Invitrogen). At the first passage, cells were collected and retrovirally transduced at MOI 1.5 using vectors that constitutively express HSV-TK or GFP transgene under control of the EFS promoter and a puromycin resistance gene. Subsequently transduced cells were enriched using $3 \mu \mathrm{g} / \mathrm{ml}$ puromycin (Sigma-Aldrich). After expansion, cells were cryopreserved at a density of $5 \times 10^{6} \mathrm{MSCs} / \mathrm{ml}$.

The cryopreserved cells were analyzed with regard to surface marker expression, differentiation capacity and transgene expression. Adipogenic and osteogenic differention were analyzed with the StemMACS Media (Miltenyi Biotech) according to the manufacturers' instructions. Transgene expression (GFP, HSV-TK) and the presence (CD73, CD90, CD105) and absence (CD19, CD34, CD45, HLA-DR) of surface marker were analyzed by flow cytometry (FC500, Beckman Coulter). After thawing the viability of MSCs was determined by Annexin V (eBioscience)/7AAD (Beckman Coulter) flow cytometry.

\section{Cell culture}

The human glioblastoma cell lines U87 (American Type Culture Collection, Manassas, VA) and NCEG55T2 [6] and the murine glioblastoma cell line GL261 
(DCTDC Tumor Repository) were cultured in MEMalpha (Invitrogen, Carlsbad, CA, USA) and Dulbecco's modified Eagle's medium (DMEM) (Invitrogen), respectively, supplemented with $2 \mathrm{mM}$ L-glutamine, $2 \mathrm{mM}$ sodium pyruvate, $100 \mathrm{U} / \mathrm{ml}$ penicillin, $100 \mu \mathrm{g} / \mathrm{ml}$ streptomycin, $0.25 \mu \mathrm{g} / \mathrm{ml}$ fungizone and $10 \%$ fetal bovine serum (Invitrogen). The multipotent neural stem cell line HB1.F3 has been extensively characterized in previous studies [16] and was maintained as adherent cultures in Dulbecco's modified Eagle medium (DMEM) supplemented with 2 mM L-glutamine and 10\% fetal bovine serum (Invitrogen). The human L87 MSC cell line [35] was cultured in RPMI (Lonza) supplemented with 10\% FBS (Biochrom) and 1\% Glutamax (Gibco). All cells were maintained in tissue culture flasks in $5 \% \mathrm{CO}_{2} / 95 \%$ air at $37^{\circ} \mathrm{C}$ in a humidified incubator and were routinely passaged at confluency. For the in vivo experiments, the cells were dispersed with a $0.05 \%$ solution of trypsin/EDTA (Invitrogen) or directly thawed, washed with phosphate-buffered saline (PBS) and adjusted to the final concentration in PBS. To obtain conditioned media, the cell lines U87, G55T2 and GL261 were grown on $35-\mathrm{mm}$ plates to $80 \%$ confluency. Cultures were washed three times with serum-free medium and incubated for another 48 hours in serum-free DMEM low glucose supplemented with $2 \mathrm{mM}$ L-glutamine. Conditioned media were collected, cleared by centrifugation for 10 minutes at $600 \mathrm{~g}$, and stored at $-80^{\circ} \mathrm{C}$. Cell labeling using the lipophilic tracer DiI (Molecular Probes, Eugene, OR, USA) was performed for 30 minutes according to the manufacturer's protocol. In vivo tracking of the human mesenchymal stem cells L87 by MR imaging was enabled by labeling the cells with superparamagnetic iron oxide particles (SPIO) as described previously [7]. Briefly, SPIO (25 mg of Fe per milliliter; Micromod, Rostock, Germany) were added to poly-L-lysine (750 ng/ml) (Sigma, Munich, Germany) and mixed with medium at room temperature for 60 minutes. The medium of cultured MSC was then replaced with the freshly prepared labeling solution and incubated for 24 hours. Cellular internalization of SPIO particles was confirmed prior to intracerebral injection by Prussian blue staining.

\section{Gap junction assay}

HSV-TK expressing MSCs (MSC_HSV-TK) were incubated with or without $100 \mu \mathrm{M}$ gap junction inhibitor Carbenoxolone (Sigma) for 16-20h before cell detachment. Cells were harvested, washed in PBS and stained with $5 \mu \mathrm{M}$ CMTPX (gap junction impermeable; Molecular Probes) and $1 \mu \mathrm{M}$ Calcein AM (gap junction permeable; Molecular Probes) for $20 \mathrm{~min}$ at $37^{\circ} \mathrm{C}$. After washing, MSCs were mixed with tumor cells at a ratio of $1: 1$ and were plated at a density of $1 \times 10^{5}$ cells/24-well. To assess cell contact independent dye transfer, $5 \times 10^{4}$ double stained MSC were seeded in the top well of a 96 transwell-plate, $5 \times 10^{4}$ non-labeled tumor cells were placed in the bottom well. Cells were analyzed by flow cytometry directly after mixing and after $4 \mathrm{~h}$ of (co-) cultivation to evaluate dye transfer of Calcein AM to unstained tumor cells.

\section{In vitro bystander assay}

HSV-TK expressing MSCs (MSC_HSV-TK) and CMFDA-stained tumor cells (U87, G55T2, GL261) or GFP-expressing U87 cells (for ratio assay) were cocultured at a ratio of $1: 1$ or the indicated ratios. In all samples the tumor cell number was kept constant at $3.1 \times 10^{4}$ per 12 well. Medium was changed to medium containing $100 \mu \mathrm{M}$ ganciclovir (GCV) on day 1, 2 and 3 after seeding. Cocultures without GCV addition and tumor cells only were used as controls. The percentage of surviving tumor cells was analyzed by flow cytometry on study day 4, after addition of counting beads (Molecular Probes).

\section{In vitro migration}

MSC migration in response to tumor-conditioned media was assessed using a modified Boyden chamber assay as previously described [15]. Briefly, quadruplicates of the chemoattractants were added to the lower wells of a 96-well modified Boyden chamber (Neuroprobe, Cabin John, MD), and wells were covered with an 8 - $\mu$ m pore size Nucleopore filter coated with $100 \mu \mathrm{g} / \mathrm{ml}$ collagen (Sigma). MSCs or HB1.F3 were then suspended at 2.5 or $1.5 \times 10^{4}$ cells in $50 \mu \mathrm{l}$ of serum-free DMEM medium containing $0.1 \%$ bovine serum albumin and seeded into the upper wells. After incubation for $5.5 \mathrm{hr}$ at $37^{\circ} \mathrm{C}$, nonmigrated cells were scraped off the upper side of the filter and filters were stained with Diff Quick (Dade, Switzerland). Nuclei of migrated cells were counted in ten high-power fields using a 20x objective with a calibrated ocular grid. Values were assessed in quadruplicate and expressed as the mean +/- standard deviation. The control migration was assessed in response to serum-free DMEM containing $0.1 \%$ bovine serum albumin only and reflects the basal migration rate of MSCs in this assay.

\section{In vivo studies}

Orthotopic glioblastoma xenografts were established in 4- to 6-week-old male NMRI-nu/nu mice (Charles River, Sulzfeld, Germany). Mice were anesthetized (100 $\mathrm{mg} / \mathrm{kg}$ ketamine and $5 \mathrm{mg} / \mathrm{kg}$ xylazine) and received a stereotactically guided injection of $2 \times 10^{5}$ human U87 glioblastoma cells into the right forebrain $(2 \mathrm{~mm}$ lateral and $1 \mathrm{~mm}$ anterior to bregma, at a $2.5 \mathrm{~mm}$ depth from the skull surface).

Ten days after tumor cell injection mice received a stereotactically guided injection of freshly dissociated 
$5 \times 10^{4}$ or $2 \times 10^{5}$ DiI-labeled, SPIO-loaded or eGFPexpressing MSCs into the left forebrain contralaterally to the growing tumor in the right forebrain and sacrificed 12, 13 or 17 days after tumor cell injection for assessment of MSC migration and tumor homing. Mice without glioma xenografts served as controls.

For therapeutic assessment freshly thawed $0.4 \times 10^{6} \mathrm{HSV}$-TK expressing MSC were stereotactically injected to the tumor region two days after tumor cell application. Systemic prodrug treatment was performed by intraperitoneal injection of $50 \mathrm{mg} / \mathrm{kg} \mathrm{GCV}$ in $100 \mu \mathrm{l}$ for five days starting four days after initial tumor cell application. Tumor volume was assessed at day 20 by MR imaging. Survival was documented and animals were sacrificed at the onset of neurological deficits or significant weight loss in accordance with federal and institutional guidelines.

All animals were sacrificed by $\mathrm{CO}_{2}$ inhalation. The brain was removed, embedded in OCT, and stored at $-80^{\circ} \mathrm{C}$ until further processed for histological analysis. All animal experiments were performed in accordance with federal and institutional guidelines and approved by the Institutional Animal Care and Use Committee.

\section{Magnetic resonance imaging (MRI)}

Tumor growth and cell migration of SPIO-labeled MSC in intracerebral U87 human glioblastoma-bearing mice was evaluated on a 7T MR imaging system (ClinScan, Bruker, Ettlingen, Germany) [7]. Mice were anesthetized with 1\% isoflurane (Baxter, Munich, Germany) in oxygen $(0.5 \mathrm{l} / \mathrm{min})$. Respiratory rates were monitored using a small animal vital sign monitor (SA Instruments Inc., Stony Brook, NY, USA). Coronal 2D T2 weighted turbo spin echo images were acquired to assess tumor location and size. Sequence parameter were: TE = $39 \mathrm{~ms}, \mathrm{TR}=2500 \mathrm{~ms}, \mathrm{BW}=250 \mathrm{~Hz} /$ pixel, turbo factor 7 , matrix $=256 \times 192, \mathrm{FOV}=20 \times 15 \mathrm{~mm}^{2}, 19$ slices, 0.4 mm slice thickness with $0.1 \mathrm{~mm}$ gap. 3D susceptibility weighted imaging (SWI) was acquired in the same orientation to detect migration of SPIO-labeled cells. Sequence parameters were: $\mathrm{TE}=7 \mathrm{~ms}, \mathrm{TR}=50 \mathrm{~ms}$, FA $=15^{\circ}, \mathrm{BW}=250 \mathrm{~Hz} /$ pixel, matrix $=192 \times 144 \times 72, \mathrm{FOV}$ $=19 \times 14 \times 7.2 \mathrm{~mm}^{3}$. Images were analyzed on a viewing task card (Syngo MR B15, Siemens, Erlangen, Germany) running on the control unit of the MR system. Tumor volumes were quantified using GraphPad Prism Software (Version 4.03).

\section{Histological analysis}

Frozen brains embedded in OCT were cut into serial $10 \mu \mathrm{m}$ sections and fixed with $4 \%$ paraformaldehyde and mounted using Citifluor AF1 Glycerol/PBS (Science Services, Munich, Germany). The whole brain of tumorbearing and control animals without brain tumors was evaluated for cellular migration and accumulation by analyzing DiI- or eGFP-positive cells in DAPI counterstained sections. Hot spots of intra- (IT) and peritumoral (PT) localized MSCs were quantified by counting the number of DiI- or eGFP-positive cells in ten randomly chosen high-power fields (1376 $\mu^{2}$ each) using a fluorescence microscope (Axioskop, Zeiss, Göttingen, Germany) and imaging Software (Axiovision AC 4.1, Zeiss). The peritumoral area (PT) was defined as a zone of $100 \mu \mathrm{m}$ distance around the tumor border. SPIOlabeled cells were detected by Prussian-Blue staining. Briefly, cryosectioned brains were incubated with freshly prepared Perls' reagent (1\% potassium ferrocyanide, 3\% hydrochloric acid, in distilled water) for 60 minutes and counterstained with neutral red.

\section{Statistical analysis}

Differences between experimental groups were calculated by student's t-test or Mann-Whitney U Test and one way or ANOVA on Ranks using sigma-plot (SystatSoftware Inc., Germany). All values were calculated as means \pm standard deviation $(\mathrm{SD})$ or median \pm standard error (SE) as indicated with a p-value $<0.05$ considered statistically significant. Kaplan-Meier survival curves were statistically analyzed using log rank significance test (Aabel NG, Gigawiz, USA).

\section{Abbreviations}

CMFDA, 5-Chlormethylfluoresceindiacetat; DAPI, 4',6-Diamidin-phenylindol; DiI, 1,1'-Dioctadecyl3,3,3',3'-tetramethylindocarbocyaninperchlorat; EFS, embryonal fyn-associated substrate; FACS, fluorescenceactivated cell sorting; GCV, ganciclovir; GFP, green fluorescent protein; GMP, good manufacturing practice; HA-tag, hemagglutinin-tag; HSV-TK, herpes simplex virus-thymidine kinase; IT, intratumoral; MR, magnetic resonance; MSC, mesenchymal stem cell; OCT, optimal cutting temperature; PT, peritumoral; SD, standard deviation; SE, standard error; SPIO, superparamagnetic iron oxide; SWI, susceptibility-weighted imaging; TRAIL, tumor necrosis factor related apoptosis inducing ligand; VEGF, vascular endothelial growth factor.

\section{Author contributions}

LD, DH, SG, FGH and NOS conceived and planned the experiments. LD, SH, DH and CLM carried out the experiments. LD, CLM and JS performed the MRI analyses. LD, SM, DH, SG, CLM, JS, CG, KL, MW, FGH and NOS contributed to the analyses and interpretation of the results. FGH and NOS took the lead in writing the manuscript. All authors provided critical feedback and helped shape the research, analyses and manuscript. 


\section{ACKNOWLEDGMENTS}

We thank Svenja Zapf for excellent technical assistance. Karen Aboody and Seung U. Kim kindly provided the HB1.F3 cell line.

\section{CONFLICTS OF INTEREST}

NOS received funding from Apceth Biopharma to perform in vivo studies. $\mathrm{DH}, \mathrm{SG}, \mathrm{CG}$ and $\mathrm{FGH}$ are employees of Apceth Biopharma.

\section{FUNDING}

German Research Foundation (DFG) to NOS (Schm 1631/5-1). NOS received funding from Apceth Biopharma to perform in vivo studies.

\section{REFERENCES}

1. Stupp R, Hegi ME, Mason WP, van den Bent MJ, Taphoorn MJ, Janzer RC, Ludwin SK, Allgeier A, Fisher B, Belanger $\mathrm{K}$, Hau P, Brandes AA, Gijtenbeek J, et al. Effects of radiotherapy with concomitant and adjuvant temozolomide versus radiotherapy alone on survival in glioblastoma in a randomised phase III study: 5-year analysis of the EORTCNCIC trial. Lancet Oncol. 2009; 10: 459-66. https://doi. org/10.1016/S1470-2045(09)70025-7.

2. Sahm F, Capper D, Jeibmann A, Habel A, Paulus W, Troost $\mathrm{D}$, von Deimling A. Addressing diffuse glioma as a systemic brain disease with single-cell analysis. Arch Neurol. 2012; 69: 523-6. https://doi.org/10.1001/archneurol.2011.2910. [PubMed]

3. Giese A, Bjerkvig R, Berens ME, Westphal M. Cost of migration: invasion of malignant gliomas and implications for treatment. J Clin Oncol. 2003; 21: 1624-36. https://doi. org $/ 10.1200 / \mathrm{JCO} .2003 .05 .063$. [PubMed].

4. Muller FJ, Snyder EY, Loring JF. Gene therapy: can neural stem cells deliver? Nat Rev Neurosci. 2006; 7:75-84. https://doi.org/10.1038/nrn1829. [PubMed].

5. Aboody KS, Brown A, Rainov NG, Bower KA, Liu S, Yang W, Small JE, Herrlinger U, Ourednik V, Black PM, Breakefield XO, Snyder EY. From the cover: neural stem cells display extensive tropism for pathology in adult brain: evidence from intracranial gliomas. Proc Natl Acad Sci U S A. 2000; 97: 12846-51. https://doi.org/10.1073/ pnas.97.23.12846. [PubMed].

6. Hansen K, Muller FJ, Messing M, Zeigler F, Loring JF, Lamszus K, Westphal M, Schmidt NO. A 3-dimensional extracellular matrix as a delivery system for the transplantation of glioma-targeting neural stem/progenitor cells. Neuro Oncol. 2010; 12: 645-54. https://doi. org/10.1093/neuonc/noq002. [PubMed].
7. Reitz M, Demestre M, Sedlacik J, Meissner H, Fiehler J, Kim SU, Westphal M, Schmidt NO. Intranasal delivery of neural stem/progenitor cells: a noninvasive passage to target intracerebral glioma. Stem Cells Transl Med. 2012; 1: 86673. https://doi.org/10.5966/sctm.2012-0045. [PubMed].

8. Nakamizo A, Marini F, Amano T, Khan A, Studeny M, Gumin J, Chen J, Hentschel S, Vecil G, Dembinski J, Andreeff M, Lang FF. Human bone marrow-derived mesenchymal stem cells in the treatment of gliomas. Cancer Res. 2005; 65: 3307-18. https://doi.org/10.1158/0008-5472. CAN-04-1874. [PubMed].

9. Portnow J, Synold TW, Badie B, Tirughana R, Lacey SF, D'Apuzzo M, Metz MZ, Najbauer J, Bedell V, Vo T, Gutova M, Frankel P, Chen M, et al. Neural Stem CellBased Anticancer Gene Therapy: A First-in-Human Study in Recurrent High-Grade Glioma Patients. Clin Cancer Res. 2017; 23: 2951-60. https://doi.org/10.1158/1078-0432. CCR-16-1518. [PubMed].

10. Trounson A, McDonald C. Stem Cell Therapies in Clinical Trials: Progress and Challenges. Cell Stem Cell. 2015; 17: 11-22. https://doi.org/10.1016/j.stem.2015.06.007. [PubMed].

11. Nowakowski A, Drela K, Rozycka J, Janowski M, Lukomska B. Engineered Mesenchymal Stem Cells as an Anti-Cancer Trojan Horse. Stem Cells Dev. 2016. https:// doi.org/10.1155/2016/4956063. [PubMed].

12. von Einem JC, Peter S, Gunther C, Volk HD, Grutz G, Salat C, Stoetzer O, Nelson PJ, Michl M, Modest DP, Holch JW, Angele M, Bruns C, et al. Treatment of advanced gastrointestinal cancer with genetically modified autologous mesenchymal stem cells - TREAT-ME-1 - a phase I, first in human, first in class trial. Oncotarget. 2017; 8: 80156-66. https://doi.org/10.18632/oncotarget.20964. [PubMed].

13. Elshami AA, Saavedra A, Zhang H, Kucharczuk JC, Spray DC, Fishman GI, Amin KM, Kaiser LR, Albelda SM. Gap junctions play a role in the 'bystander effect' of the herpes simplex virus thymidine kinase/ganciclovir system in vitro. Gene Ther. 1996; 3: 85-92.

14. Matuskova M, Hlubinova K, Pastorakova A, Hunakova L, Altanerova V, Altaner C, Kucerova L. HSV-tk expressing mesenchymal stem cells exert bystander effect on human glioblastoma cells. Cancer Lett. 2010; 290: 58-67. https:// doi.org/10.1016/j.canlet.2009.08.028. [PubMed].

15. Schmidt NO, Przylecki W, Yang W, Ziu M, Teng Y, Kim SU, Black PM, Aboody KS, Carroll RS. Brain tumor tropism of transplanted human neural stem cells is induced by vascular endothelial growth factor. Neoplasia. 2005; 7 : 623-9. https://doi.org/10.1593/neo.04781. [PubMed].

16. Aboody KS, Najbauer J, Metz MZ, D'Apuzzo M, Gutova M, Annala AJ, Synold TW, Couture LA, Blanchard S, Moats RA, Garcia E, Aramburo S, Valenzuela VV, et al. Neural stem cell-mediated enzyme/prodrug therapy for glioma: preclinical studies. Sci Transl Med. 2013; 5: 184ra59. https://doi.org/10.1126/scitranslmed.3005365. [PubMed]. 
17. Aboody KS, Najbauer J, Danks MK. Stem and progenitor cell-mediated tumor selective gene therapy. Gene Ther. 2008; 15: 739-52. https://doi.org/10.1038/gt.2008.106. [PubMed].

18. Ullah M, Liu DD, Thakor AS. Mesenchymal Stromal Cell Homing: Mechanisms and Strategies for Improvement. iScience. 2019; 15: 421-38. https://doi.org/10.1016/j. isci.2019.05.004. [PubMed].

19. Schichor C, Birnbaum T, Etminan N, Schnell O, Grau S, Miebach S, Aboody K, Padovan C, Straube A, Tonn JC, Goldbrunner R. Vascular endothelial growth factor A contributes to glioma-induced migration of human marrow stromal cells (hMSC). Exp Neurol. 2006; 199: 301-10. https://doi.org/10.1016/j.expneurol.2005.11.027. [PubMed].

20. Sun L, Lee J, Fine HA. Neuronally expressed stem cell factor induces neural stem cell migration to areas of brain injury. J Clin Invest. 2004; 113: 1364-74. https://doi. org/10.1172/JCI200420001. [PubMed].

21. Schmidt NO, Koeder D, Messing M, Mueller FJ, Aboody KS, Kim SU, Black PM, Carroll RS, Westphal M, Lamszus K. Vascular endothelial growth factor-stimulated cerebral microvascular endothelial cells mediate the recruitment of neural stem cells to the neurovascular niche. Brain Res. 2009; 1268: 24-37. https://doi.org/10.1016/j. brainres.2009.02.065. [PubMed].

22. Yong RL, Shinojima N, Fueyo J, Gumin J, Vecil GG, Marini FC, Bogler O, Andreeff M, Lang FF. Human bone marrow-derived mesenchymal stem cells for intravascular delivery of oncolytic adenovirus Delta24-RGD to human gliomas. Cancer Res. 2009; 69: 8932-40. https://doi. org/10.1158/0008-5472.CAN-08-3873. [PubMed].

23. Balyasnikova IV, Prasol MS, Ferguson SD, Han Y, Ahmed AU, Gutova M, Tobias AL, Mustafi D, Rincon E, Zhang L, Aboody KS, Lesniak MS. Intranasal delivery of mesenchymal stem cells significantly extends survival of irradiated mice with experimental brain tumors. Mol Ther. 2014; 22: 140-8. https://doi.org/10.1038/mt.2013.199. [PubMed].

24. Da Mesquita S, Fu Z, Kipnis J. The Meningeal Lymphatic System: A New Player in Neurophysiology. Neuron. 2018; 100: 375-88. https://doi.org/10.1016/j.neuron.2018.09.022. [PubMed].

25. Huang Q, Liu XZ, Kang CS, Wang GX, Zhong Y, Pu PY. The anti-glioma effect of suicide gene therapy using BMSC expressing HSV/TK combined with overexpression of $\mathrm{Cx} 43$ in glioma cells. Cancer Gene Ther. 2010; 17: 192-202. https://doi.org/10.1038/cgt.2009.64. [PubMed].

26. Lee DH, Ahn Y, Kim SU, Wang KC, Cho BK, Phi JH, Park IH, Black PM, Carroll RS, Lee J, Kim SK. Targeting rat brainstem glioma using human neural stem cells and human mesenchymal stem cells. Clin Cancer Res. 2009; 15: 4925-34. https://doi.org/10.1158/1078-0432.CCR-083076. [PubMed].

27. Friedenstein AJ, Chailakhjan RK, Lalykina KS. The development of fibroblast colonies in monolayer cultures of guinea-pig bone marrow and spleen cells. Cell Tissue Kinet. 1970; 3: 393-403. https://doi. org/10.1111/j.1365-2184.1970.tb00347.x. [PubMed].

28. Mushahary D, Spittler A, Kasper C, Weber V, Charwat V. Isolation, cultivation, and characterization of human mesenchymal stem cells. Cytometry A. 2018; 93: 19-31. https://doi.org/10.1002/cyto.a.23242. [PubMed].

29. de Melo SM, Bittencourt S, Ferrazoli EG, da Silva CS, da Cunha FF, da Silva FH, Stilhano RS, Denapoli PM, Zanetti BF, Martin PK, Silva LM, dos Santos AA, Baptista LS, et al. The Anti-Tumor Effects of Adipose Tissue Mesenchymal Stem Cell Transduced with HSV-Tk Gene on U-87-Driven Brain Tumor. PLoS One. 2015; 10: e0128922. https://doi. org/10.1371/journal.pone.0128922. [PubMed].

30. Zhang J, Huang X, Wang H, Liu X, Zhang T, Wang Y, Hu D. The challenges and promises of allogeneic mesenchymal stem cells for use as a cell-based therapy. Stem Cell Res Ther. 2015; 6: 234. https://doi.org/10.1186/s13287-0150240-9. [PubMed].

31. Westphal M, Yla-Herttuala S, Martin J, Warnke P, Menei P, Eckland D, Kinley J, Kay R, Ram Z, Group AS. Adenovirusmediated gene therapy with sitimagene ceradenovec followed by intravenous ganciclovir for patients with operable high-grade glioma (ASPECT): a randomised, open-label, phase 3 trial. Lancet Oncol. 2013; 14: 823-33. https://doi.org/10.1016/S1470-2045(13)70274-2.

32. Wang XJ, Xiang BY, Ding YH, Chen L, Zou H, Mou XZ, Xiang C. Human menstrual blood-derived mesenchymal stem cells as a cellular vehicle for malignant glioma gene therapy. Oncotarget. 2017; 8: 58309-21. https://doi. org/10.18632/oncotarget.17621. [PubMed].

33. Ehtesham M, Kabos P, Kabosova A, Neuman T, Black KL, Yu JS. The use of interleukin 12-secreting neural stem cells for the treatment of intracranial glioma. Cancer Res. 2002; 62: 5657-63.

34. Martinez-Quintanilla J, Bhere D, Heidari P, He D, Mahmood U, Shah K. Therapeutic efficacy and fate of bimodal engineered stem cells in malignant brain tumors. Stem Cells. 2013; 31: 1706-14. https://doi.org/10.1002/ stem.1355. [PubMed].

35. Thalmeier K, Dörmer P. Stromal cell lines from human bone marrow and their use. United States Patent 5,658,761. 1997. 